\title{
Method of increasing spatial resolution of the scanning near-field microwave microscopy
}

\author{
R. Kantor ${ }^{\mathrm{a})}$ and I. V. Shvets \\ SFI Laboratories, Department of Physics, Trinity College, Dublin 2, Ireland
}

(Received 6 June 2002; accepted 25 September 2002)

\begin{abstract}
In this article we propose methods for the measurement of electric intensity of a microwave field above the surface of microwave circuits. Using miniaturized coaxial antennas and a special probe positioning system, we measure both the amplitude and the phase of the induced field above the device under test. We introduce a position/signal difference method to further increase the spatial resolution down to about $30 \mu \mathrm{m}$-about one order better than contemporary microwave scanning devices utilizing coaxial antennas. The effect is theoretically analyzed and experimentally verified. The probes are calibrated in a well-defined field standard to allow quantitative characterization of the measured field. Performance of our scanning system utilizing these methods is demonstrated using a PCB finger capacitor. (C) 2003 American Institute of Physics. [DOI: 10.1063/1.1522486]
\end{abstract}

\section{INTRODUCTION}

Contemporary systems for inspection of microwave circuits are usually based on measurement of the signals at the device ports. In most cases they are limited to the measurement of the input and output signals. Such measurements are often not sufficient for characterizing the functionality of individual elements and do not give a full description of distribution of circuit signals. In some cases special measurement pads within the system are designed for direct coupling of the probes. Unfortunately, their use is limited due to relatively large contact areas, their influence on the circuit properties caused by their high capacitance to the ground, cross coupling with other circuit elements and also the additional load of the measuring probes. These drawbacks effectively eliminate the use of such measurement techniques in highly miniaturized circuits, especially in microwave monolithic integrated circuits (MMIC). Due to the abovementioned reasons, noncontact scanning near-field measurements may become an attractive method for testing circuit performance and failure analysis. By analyzing the field distribution above the circuit surface one can evaluate not only values of the field sources (charges/potentials, currents) ${ }^{1,2}$ but also signal coupling between circuit parts, electromagnetic emission of the components of the device and other aspects of electromagnetic compatibility.

\section{ELECTRIC FIELD ANTENNAS}

For acquisition of microwave intensities in a near-field region, short monopoles ${ }^{1,3}$ or magnetic loops ${ }^{4,5}$ can be used for electric and magnetic field component detection, respectively. We have focused our attention on the measurement of electric intensity using miniaturized coaxial antennascylindrical short monopoles where a central conductor protrudes for a defined length from the shielding (see Fig. 1). Our antennas are vertically positioned and, due to their axial

${ }^{a)}$ Electronic mail: rkantor@tcd.ie symmetry, they are sensitive to the normal component of the electric field intensity. The length of the protruding conductor must not exceed the desired spatial resolution and in many cases it is chosen to be comparable or shorter than the diameter $D$ of the shielding. Unfortunately, the antenna's resolution does not just depend on the length of the protruding conductor but also on the diameter of the shielding surrounding it. Surface currents within the shielding also induce a secondary field and change the input signal. For welldefined antenna geometry the signal level can be evaluated using numerical simulation methods such as finite-difference time domain. As the result of the simulation depends on the particular field distribution, the external field is commonly assumed to be homogeneous, thus resulting in a single sensitivity coefficient, that is the ratio between the signal level and the field intensity. ${ }^{3}$ When the field is highly concentrated around the apex of the protruding conductor, images with spatial contrast to a certain degree better than the length of the conductor and the dimensions of the shielding can be obtained. Unfortunately, those images lack good quantitative characterization as the antenna's signal level depends on particular distribution of the field that can no longer be considered to be homogeneous. Additionally, the presence of the shielding close to the circuit may cause redistribution of the charges in the circuit and distortion of the primary field $E^{p}$.

\section{POSITION/SIGNAL DIFFERENCE METHOD}

It appears that only by decreasing the antenna dimensions along with coaxial shielding its spatial resolution capability can be improved. Unfortunately, miniaturization of the antenna to the micrometer range makes its fabrication rather difficult, especially fabrication of the coaxial line of low diameter and the forming of a short protruding central conductor. In this article we present a scanning method which overcomes the resolution limit determined by the antenna's dimensions and allows us to increase its resolution capability without the need for further miniaturization of the antenna. Our antennas have a shield with an outer diameter of $D=230$ 


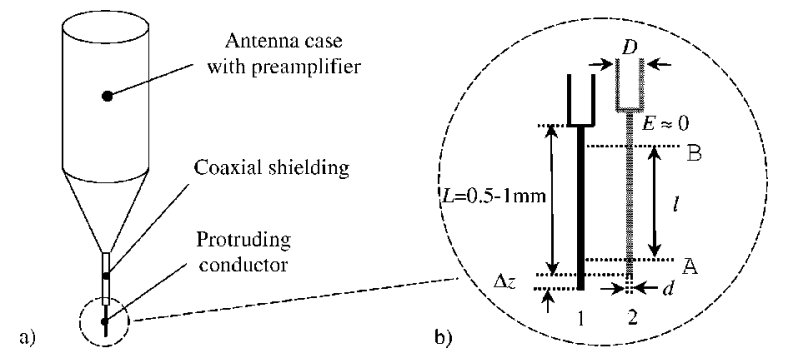

FIG. 1. Electric field probe: (a) outline, (b) active protruding conductor. The two positions of the antenna corresponding to different heights above the surface of the device under test are indicated in figure (b) by numbers 1 and 2 .

$\mu \mathrm{m}$ and a relatively long and thin central protruding conductor: a copper wire of length $L \simeq 0.5-1 \mathrm{~mm}, L \gg d, d$ $=8 \mu \mathrm{m}$ as shown in Fig. 1(b). The measurement method is based on comparing results of two subsequent scans with the antenna displaced by a small distance along its axis. We will show that for high density structures, when most of the field gradient is located close to the surface of the structure, the signal difference is determined only by the field strength surrounding the apex of the central conductor. By subtracting the two signals corresponding to two positions of the antenna displaced along its axis by distance $\Delta z$, one can cancel the contribution of the field in middle section of the protruding conductor, between planes $\mathcal{A}$ and $\mathcal{B}$ in the Fig. 1(b). Because for high-density structures the field above that region is supposed to be negligible, in this way the field surrounding the conductor apex can be isolated and measured. We shall call this method position/signal difference and we will show that it allows improvement of the spatial resolution of mapping of the microwave field.

\section{ANALYSIS OF THE METHOD}

The antenna displacement is equivalent to changes in its geometry: reduction of the length of the protruding conductor by $\Delta z$ and displacement of the front end of the shield by the same value. In order to show that the contribution of the external field from the middle section of the antenna can be suppressed, we will analyze the influence of the field and distribution of the currents induced in that region. Thanks to axial symmetry of the antenna, the analysis can be reduced to investigating the boundary condition at the center of the conductor. As the sum of the primary incident electric field and the secondary electric field induced by the antenna surface currents must vanish inside the conductor, the condition for longitudinal component of the electric field (parallel to the antenna axis) before and after displacement of the antenna can be written as

$$
\begin{aligned}
& E_{z}^{p}(z)+\int G_{1}\left(z, z^{\prime}\right) J_{1}(z) d z^{\prime}=0, \\
& E_{z}^{p}(z)+\int G_{2}\left(z, z^{\prime}\right) J_{2}(z) d z^{\prime}=0 .
\end{aligned}
$$

Here the primary field at the conductor center $E^{p}(z)$ and its $z$-component $E_{z}^{p}(z)$ do not depend on the probe position. The integrals in Eqs. (1) and (2) represent the secondary field induced by the antenna as a sum of the contributions of the surface current elements to the $z$ component of the electric intensity. The integrals are taken over the entire current space. $J_{1}(z)$ and $J_{2}(z)$ signify surface currents before and after the displacement, respectively. The Green functions $G_{1,2}\left(z, z^{\prime}\right)$ represent the weighting coefficients of the contribution of current sources to the secondary field. For the protruding cylindrical conductor the coefficient does not change as a result of displacement along its axis as it depends only on the position of the observation point $z$ relative to the position of the current source $z^{\prime}$

$$
G_{1}\left(z, z^{\prime}\right)=G_{2}\left(z, z^{\prime}\right)=G\left(z-z^{\prime}\right) .
$$

Its explicit form is ${ }^{6}$

$$
\begin{aligned}
G\left(z-z^{\prime}\right)= & \frac{-i Z_{0} \exp (-i \beta r)}{\beta 4 \pi r^{3}} \\
& \times\left[(1+i \beta r)\left(2-\frac{3}{4} \frac{d}{r}\right)+\beta^{2} \frac{d^{2}}{4}\right],
\end{aligned}
$$

where $Z_{0}$ is the impedance of vacuum, $d$ is the diameter of the conductor, $r=\sqrt{\left(z-z^{\prime}\right)^{2}+d^{2} / 4}$ is the distance between the current source and the observation point at the axis of the conductor and $\beta=2 \pi / \lambda$ is the propagation constant.

Let us focus our attention on the middle section of the protruding conductor in order to show that in that section the difference between the currents before and after the displacement is determined only by the currents at its boundaries and, therefore, the influence of the external field on the current difference can be removed. The section is defined by the planes $\mathcal{A}$ and $\mathcal{B}$ in between which the secondary field, induced by the antenna currents, can be calculated as a result of contribution of currents within the protruding conductor, excluding the displaced apex and also excluding the contribution from the currents in the shielding. Because for $r$ $\gg \frac{3}{8} d$ the Green function (4) decays proportionally to $r^{-3}$ and the integrals (1), (2) quickly converge, the distances to the plane $\mathcal{A}$ from the antenna displaced apex and the shielding can be chosen greater than diameter $d$ so that the secondary induced field in this section is not directly influenced by the currents induced in the displaced apex. For the same reason the separation between the plane $\mathcal{B}$ and the shielding can be chosen greater than diameter $D$ so that the contribution of the shielding currents to the field induced inside the section can be neglected. The integration space can therefore be effectively limited to the length $L$ of the central conductor. By subtracting (1), and (2) and by taking into account (3) we get a single equation for the difference of currents in that region

$$
\int_{L} G\left(z-z^{\prime}\right) \mathcal{J}(z) d z^{\prime}=0
$$

where $\mathcal{J}(z)=J_{1}(z)-J_{2}(z)$. The solution of (5) for current difference distribution in that region is not dependent on the external field. Since above plane $\mathcal{B}$ the strength of the external field is assumed to be negligible, the difference of the currents $J_{z}$ is influenced only by the local field below plane $\mathcal{A}$ that surrounds the displaced antenna apex. This difference depends on the virtual changes in its geometry and changes 
in the boundary conditions in the presence of the external electric field. As the virtual changes are limited to the region $\Delta z$ of the displaced antenna apex, the measured signal and the resolution of the measurement method is determined by the displacement $\Delta z$.

Although most of the protruding conductor acts only as a transmitter between the displaced apex and the input to the coaxial line, its length may have an effect on the efficiency of signal matching and the level of measured signal. We therefore describe in detail the influence of this length on the transmission of the signal between planes $\mathcal{A}$ and $\mathcal{B}$. The integral Eq. (5) resembles the boundary condition for the uniform transmission line with no induced longitudinal electric field component. Its only nontrivial solution can be written in the form of two sinusoidal waves traveling in the two opposite directions

$$
\mathcal{J}(z)=\mathcal{J}_{1} \exp (-i \beta z)+\mathcal{J}_{2} \exp (i \beta z) .
$$

The current amplitude constants $\mathcal{J}_{1}, \mathcal{J}_{2}$ must match the field solution for the antenna apex below plane $\mathcal{A}$ and at the input to the shielding above plane $\mathcal{B}$. We know that below plane $\mathcal{A}$ the currents depend on the field surrounding the displaced antenna apex and if we choose the origin for the $z$ axis at the plane $\mathcal{A}\left(z_{A}=0\right)$, the current difference $\mathcal{J}_{\mathcal{A}}$ can be written in accordance with Eq. (6)

$$
\mathcal{J}_{\mathcal{A}}=\mathcal{J}_{1}+\mathcal{J}_{2} \text {. }
$$

The solution for the currents at the input to the coaxial line without a presence of the external field depends only on its geometry. It can be expressed in terms of the reflection coefficient $h_{11}$ for the currents at plane $\mathcal{B}$ for which

$$
h_{11}=\frac{\mathcal{J}_{2} \exp (i \beta l)}{\mathcal{J}_{1} \exp (-i \beta l)} \text {. }
$$

In principle, the displacement of the front end of the shield should result in change in the value of $h_{11}$. However, as $\Delta z$ is negligibly small by comparison with wavelength $\lambda$, such a change in the geometry of the coaxial input can be neglected and $h_{11}$ can be assumed constant. In practice $h_{11}$ is close to unity due to a large mismatch between relatively low input impedance of the coaxial line and very high impedance of the free conductor, determined by its residual coupling to the shielding. Using Eqs. (6), (7) and (8) the transfer function of current difference from the apex to the input of the coaxial line can be written as

$$
\mathcal{J}_{\mathcal{B}}=\exp (-i \beta l) \frac{1+h_{11}}{1+h_{11} \exp (-2 i \beta l)} \cdot \mathcal{J}_{\mathcal{A}} .
$$

It may seem that the length of the protruding conductor can be adjusted for optimum signal matching at $l \simeq \lambda / 4$ for which the antenna operates at its resonance. Unfortunately, the mechanical properties of the conductor do not allow for extension of the length $L$ above 1-2 $\mathrm{mm}$. The vibrations and lateral bending of such a long and thin wire, mostly caused by air flow fluctuations and accelerations during scanning movement, cause degradation of the resolution. For our frequencies of interest $(1-8 \mathrm{GHz})$ this length is significantly below $\lambda / 4$ and the magnitude of the transfer function (9) is close to unity

$$
J_{B} \simeq J_{A} .
$$

\section{SENSITIVITY OF THE SYSTEM}

The above analysis shows that one can improve the resolution by reducing the displacement $\Delta z$. At the same time this leads to reduction in the signal level. As the signal level must exceed the noise level, this may effectively limit the resolution of the antenna and make it dependent on minimal detectable field intensities. The level of acquired signal depends not only on the currents induced in the apex of the conductor but also on the efficiency of its matching to the input of the coaxial line, the properties of the preamplifier and the transmission of the signal to the acquisition system - to a vector network analyzer (VNA). The apex of the protruding conductor functions as a near ideal current source and one of the main factors influencing the sensitivity is the matching of such a high-impedance source to the input of the coaxial line and subsequently to a preamplifier. For the antenna active region, corresponding to displacement $\Delta z$ of 5-50 $\mu \mathrm{m}$, the impedance has capacitive character of values above $10^{4} \Omega$. Because the transfer function (10) preserves the high impedance character of the apex current source, to improve the matching efficiency the input impedance of the coaxial line has to be increased. The mismatch, compared to standard transmission lines and amplifiers with impedances of about $50 \Omega$, is in the range $10^{3}-10^{4}$ and therefore microwave resonators (microwave cavities, coaxial resonators ${ }^{7,8}$ ) with high quality factor have to be applied to optimize the signal matching. Unfortunately, their dimensions and mass do not allow their incorporation into our probes; furthermore, their narrow resonance response would limit the bandwidth of the transmitted signal.

For our miniature probes we have chosen a simpler matching scheme which uses a quarter-wavelength transformer formed by the antenna coaxial input line with a relatively high characteristic impedance $Z_{c} \simeq 120 \Omega$. By choosing the length of this line to be equal to $\lambda / 4$ the impedance at the input can be increased to the value $Z_{i}=Z_{c}^{2} / Z_{50}$ where $Z_{50}$ is the input impedance of the subsequent network-in our case a MMIC preamplifier. For the frequency of interest (4 $\mathrm{GHz}$ ) the sensitivity increase resulting from this matching procedure was about $15 \mathrm{~dB}$.

\section{CALIBRATION OF THE PROBES}

As the properties of antennas may vary, each antenna must be individually calibrated in a well-defined field standard. We have calibrated our antennas in the nonhomogeneous field above an air-suspended cylindrical transmission line (see Fig. 2) which allows good access for the probes and for which the surrounding field can be explicitly calculated. As for the position/signal difference method, the active antenna region corresponds to the displaced apex of the conductor; the level of the resulting signal difference depends on the displacement value. Both mathematical simulations and the experimental results (Fig. 3) give a highly linear character of this dependency. As a typical preamplifier is also highly linear, the measured voltage $U$ after its conditioning and transmission to the input of the acquisition instrument 

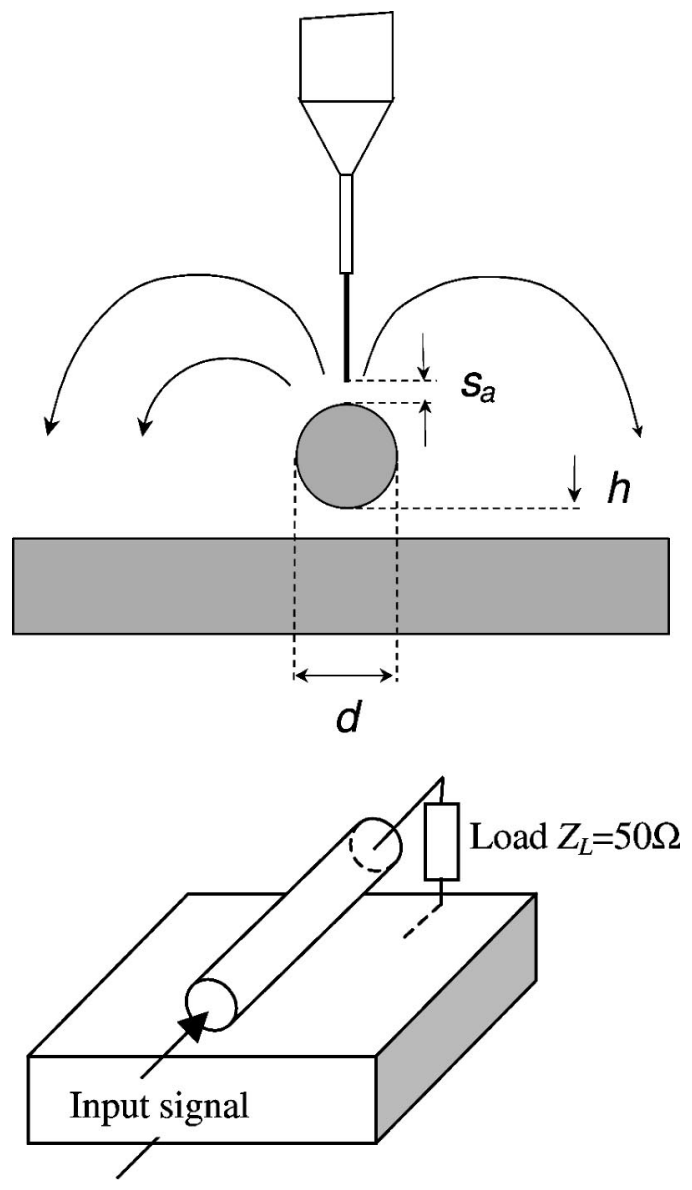

FIG. 2. Calibration unit: an air-suspended transmission line with characteristic impedance of $50 \Omega(d=1.25 \mathrm{~mm}, h=0.23 \mathrm{~mm})$ is terminated by a load of the same impedance to avoid signal reflection. The field at any coordinate can be calculated if the power coupled to the unit is known.

(VNA) is proportional to the antenna displacement $\Delta z$. We can, therefore, define the sensitivity of the system for a particular frequency by a single unit-less constant $S$

$$
S=\frac{1}{\Delta z} \frac{U}{E_{z}} .
$$

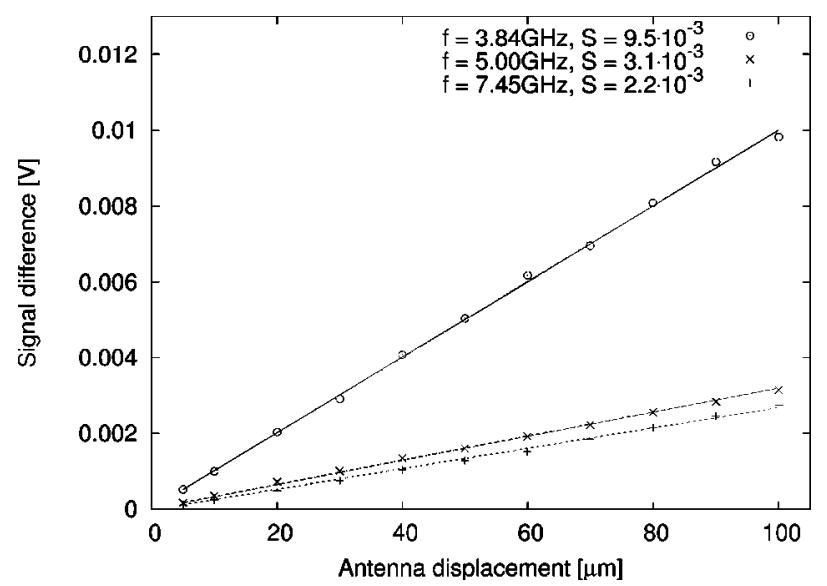

FIG. 3. Linear dependency of measured signal difference $U$ on antenna displacement $\Delta z$. The design of the antenna was optimized for higher sensitivity $S$ at frequencies close to $4 \mathrm{GHz}$.

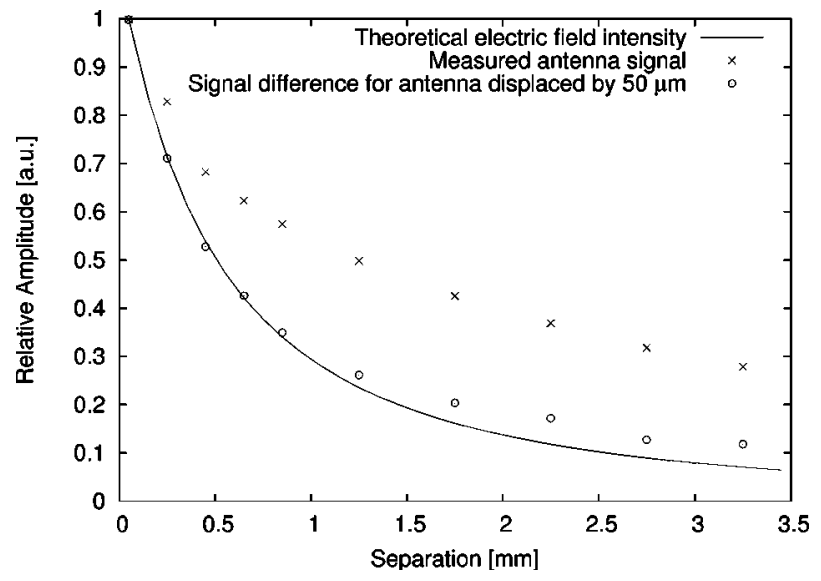

FIG. 4. Calculated electric field intensity and measured antenna signals above an air suspended cylindrical transmission line shown in Fig. 2 for various separation $s_{a}$ between the antenna apex and the line.

Here $E_{z}$ is the vertical component of electric field intensity of microwave field. This constant, determined from such calibration measurement, can then be used during the scanning process for the calculation of real values of the electric field. The measured sensitivity constant gives us minimum level of detectable electric field intensity of about $15 \mathrm{~V} / \mathrm{m}$ for displacement $20 \mu \mathrm{m}$ (and comparable spatial resolution) with noise signal level of about $-95 \mathrm{dBm}$ for bandwidth 10 $\mathrm{Hz}$ at $4 \mathrm{GHz}$ frequency.

\section{EXPERIMENTAL VERIFICATION OF THE METHOD}

To verify the position/signal difference method we have compared expected field values in the calibration unit shown in Fig. 2 with the measured data. Figure 4 shows signal values for different separation $s_{a}$ between the antenna and the transmission line. All signal curves were normalized to the values corresponding to the antenna apex placed close to the cylindrical conductor of the calibration unit with the separation of $50 \mu \mathrm{m}$ from the signal line. We see that direct signal, as acquired by the antenna, does not compare directly to the field strength as the field intensity changes along the active protruding conductor and the signal level depends on the particular distribution of the field. On the other hand, the curve of signal difference, with antenna displacement by $\Delta x=50 \mu \mathrm{m}$ follows faithfully expected field intensity at the apex of the protruding conductor. We can observe very good agreement between those curves, especially for distances smaller than $1 \mathrm{~mm}$ where high field gradient is expected. This agreement is noticeably worse for greater distances, mostly caused by limited dimensions of the calibration unit and distortion of the field at greater distances from the cylindrical conductor.

\section{PROBE POSITIONING SYSTEM}

Our goal was to achieve field images with spatial resolution of $R$ about $10^{-5} \mathrm{~m}$. For this the antenna must be driven with a precision better than the desired resolution $R$. We use a combination of precision motorized positioning stages and piezo actuators which allows us to scan large areas of up to several $\mathrm{cm}$ with high dynamics of probe mo- 


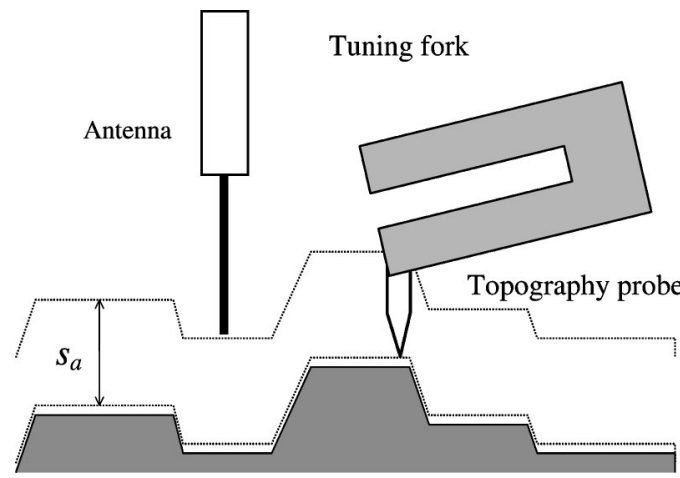

FIG. 5. Separate topography and field acquisition during the scanning process.

tion and allows us to keep submicron probe positioning accuracy. The movement of the antenna over the sample surface has to be accurately controlled as the antenna has to be driven very close to the circuit surface with a separation corresponding to the desired resolution. Conventional horizontal plane scanning ${ }^{1,3,9}$ cannot be implemented with sufficient precision for highly miniaturized circuits if the small tip sample separation $s_{a}$ has to be kept constant. This is due to the sample tilt and also because the surfaces of most circuits are not flat: step-like profile of the transmission lines and additional surface features like air bondings, signal contacts or shunt elements are typically comparable or else exceed the required working distance. Methods utilizing simultaneous tip/sample distance control techniques such as scanning tunneling microscopy (STM), ${ }^{10}$ capacitive distance control using dual frequency excitation ${ }^{11}$ or incorporation of a microwave feedback for distance control ${ }^{12,13}$ are applicable for conductive samples only and their use is limited to sample profile mapping and studies of properties of materials. $^{14,15}$
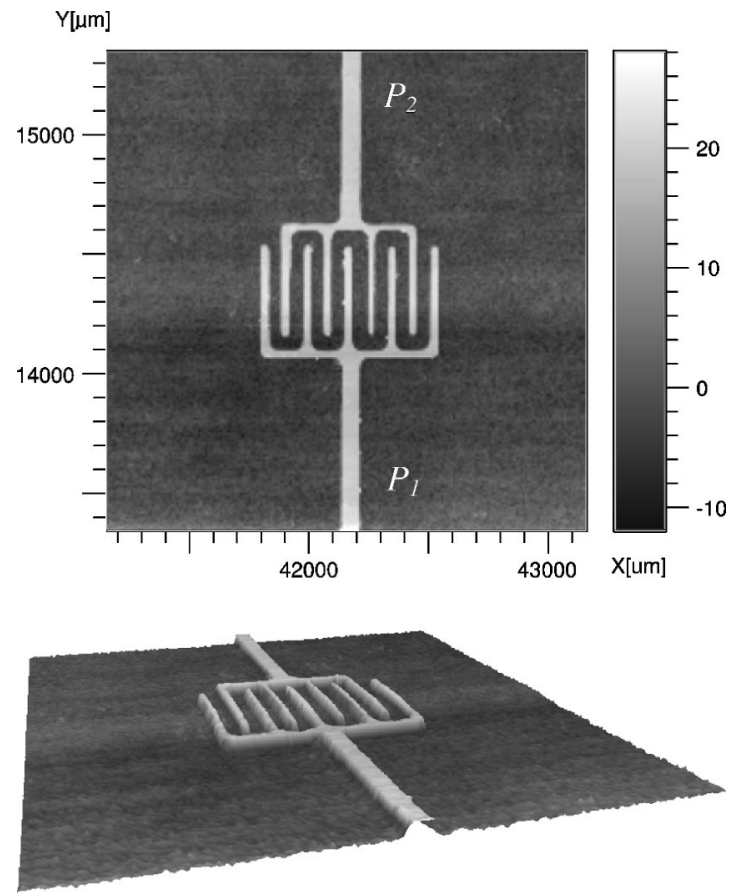

FIG. 6. Topography of the PCB surface capacitor. Thickness of the dielectric substrate is $t=127 \mu \mathrm{m}$, permittivity $\epsilon=10.2$. The width of the fingers is $40 \mu \mathrm{m}$, separation gap between the fingers is about $60 \mu \mathrm{m}$.

Our measurement process consists of two separate steps: sample topography acquisition and field probe scanning. During the second step the antenna is driven according to previously acquired topographic data (see Fig. 5) at a defined distance above the surface. This allows choosing an arbitrary separation between the front end of the antenna and the surface and keeping it constant during the field measurements. The topography acquisition is performed using an atomic force microscopy-like technique where a glass probe is dith-
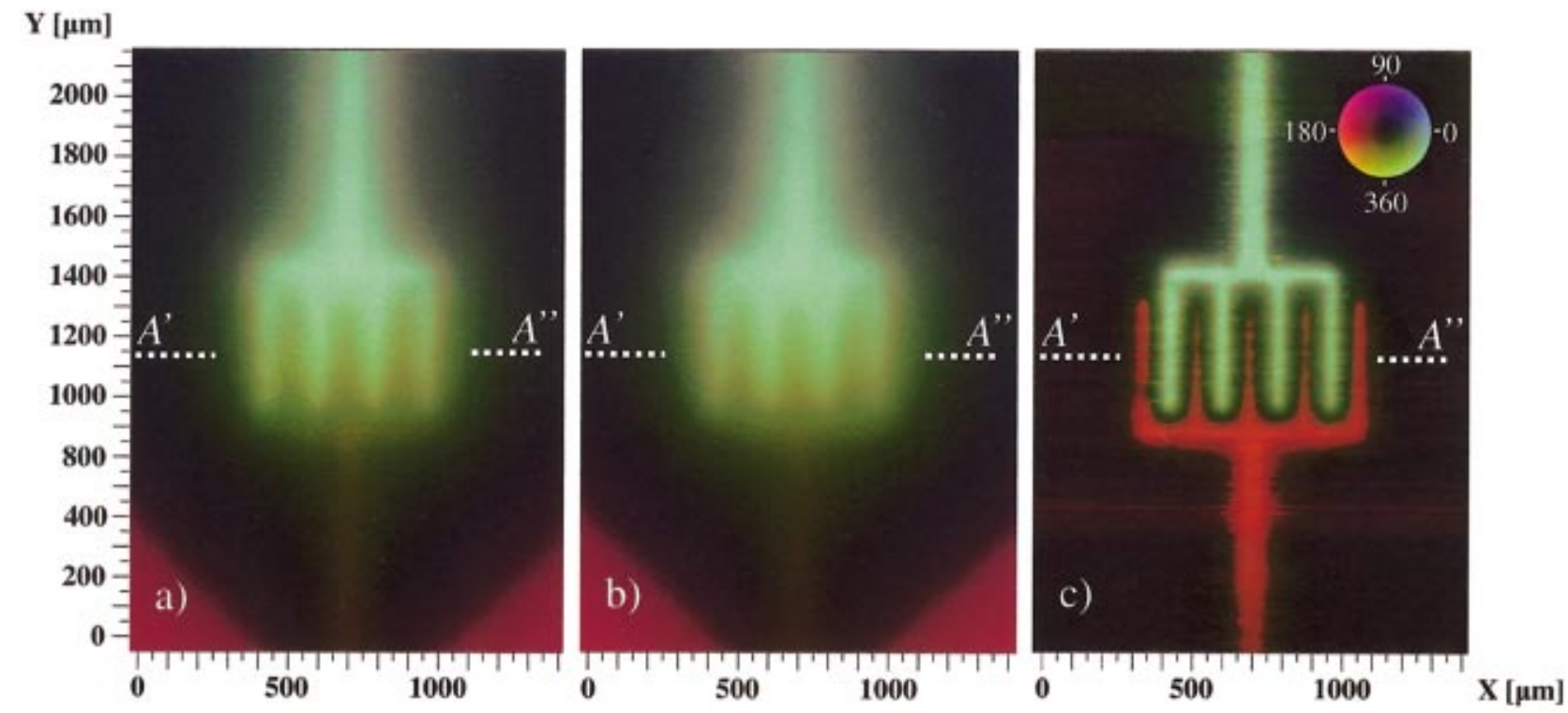

FIG. 7. (Color) Effect of resolution enhancement as a result of signal differences acquired for different antenna/sample separation $s_{a}$. The images represent both the amplitude and the phase of the signal. The amplitude of the acquired signal corresponds to the brightness in the image, the phase is expressed by the color as indicated by the ring scale in figure (c). (a) Signal induced in the antenna for separation $s_{a}=5 \mu \mathrm{m}$, (b) signal induced in the antenna for separation $s_{a}=12 \mu \mathrm{m}$, (c) Difference of signals (a) and (b) which suppress background fields acting along the protruding conductor above its displaced apex. 
ered perpendicular to the surface. The dependency of the amplitude and the phase of the probe's mechanical oscillation on the probe/sample separation is used to keep the separation constant in the range of several tens of $\mathrm{nm}$. A quartz tuning fork, commonly used in scanning near-field optical microscopy ${ }^{16,17}$ in a single oscillating arm configuration ${ }^{18}$ is utilized for probe frequency stabilization and amplitude detection. The technique can be employed with all types of materials used in circuit fabrication to include various metals, dielectrics and semiconductors. After the topography acquisition, the probe is exchanged for the field antenna and field scanning for various separations between the antenna's apex and the surface is performed. A static reference tip is used when the topography probe is exchanged for the antenna; the probes are aligned by means of an optical control using a long-focal length microscope.

\section{SCANNING RESULTS}

A surface capacitor was used to demonstrate the method described. It was fabricated by us using the standard lithographic process on a microwave PCB sheet as seen in the Fig. 6. The signal from the source of VNA is coupled to the port $P_{1}$ of the capacitor. Port $P_{2}$ is terminated by a shortcut at a distance of about $25 \mathrm{~mm}$ from the capacitor to allow excitation of the capacitor to higher potentials due to resonance in the transmission line at the frequency of interest $f$ $=3.84 \mathrm{GHz}$. Both the amplitude and the phase of the signal are acquired by the antenna and stored during the scanning process.

In Figs. 7(a) and7(b) we can see field images of the normal electric field acquired for two different antenna/ sample separations; Fig. 7(c) shows the difference of these signals. We can clearly observe significant resolution enhancement for the difference signal. As the antenna is sensitive to the field acting along the whole length of the protruding conductor, the scattered field intensities at higher distances above the sample represent the main contribution to the level of acquired signal. The difference signal corresponds to the local electric field intensities surrounding the antenna apex only. The method also reveals the differences in the phase of electric field; these differences are indicated by different colors in the field map inserted in Fig. 7(c). The amplitude and the phase of electric field intensities are directly determined by alternating potentials of the fingers belonging to the two different ports $P_{1}$ and $P_{2}$ of the capacitor.

The resolution enhancement is also illustrated in Fig. 8 (a) which represents cross-section $A^{\prime}-A^{\prime \prime}$ of the signal amplitude across the fingers of the capacitor. Figure 8(b) exposes the phase differences of about $55^{\circ}$. For original fulllength antenna signals, the phase changes across the image are nearly indistinguishable. The increase in the phase contrast is possible due to the fact that the difference is calculated by subtracting complex amplitudes of the signals. Indeed the vector difference between two nearly identical vectors can have phase completely different from the phases of such vectors. In other words the phase contrast came from the fact that although the phase of the measured field does not change at significant heights above the surface, it does
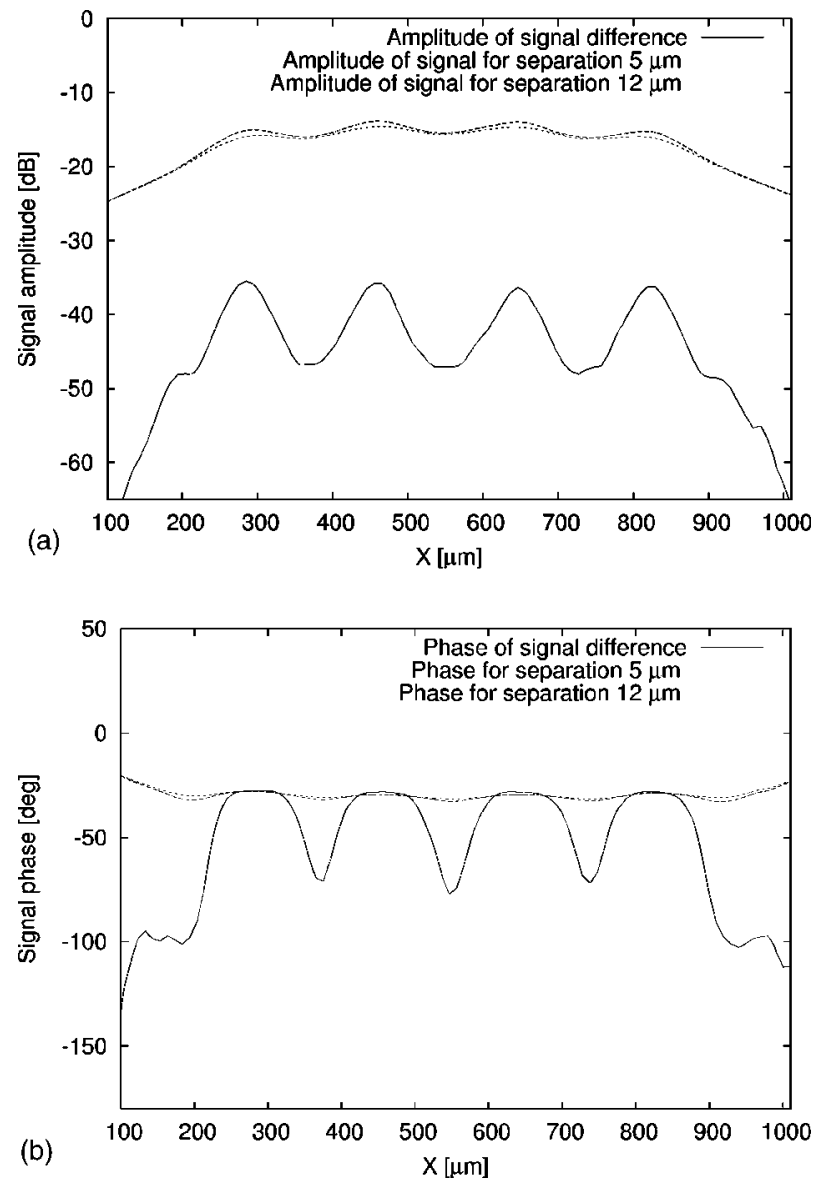

FIG. 8. Increase in spatial contrasts for cross-section it $A^{\prime}-A^{\prime \prime}$ using position/signal difference method. The method reveals the difference of about $12 \mathrm{~dB}$ in the amplitude and $55^{\circ}$ in the phase of local fields surrounding different fingers of the capacitor. (a) Amplitude of the signals, (b) phase of the signals.

change for small separations above the circuit which is represented by the signal difference.

The analysis of the Figs. 7 and 8 would suggest that the spatial resolution obtained by position/signal difference method is better than $30 \mu \mathrm{m}$. This gives the ratio of the resolution to the wavelength $R / \lambda$ of some $3 \times 10^{-4}$.

\section{CONCLUSIONS}

In order to measure electric field intensities with high resolution, a scanning setup combining topography and microwave field acquisition was developed. We have presented miniaturized field probes and measurement techniques allowing acquisition of electric field intensity in the deep nearfield region $\left(\lambda / 10^{3}-\lambda / 10^{4}\right)$. In particular, the position/signal difference method appears to be an effective approach allowing one to achieve exceptional resolution with low distortion of the measured field and good quantitative field characterization. Although we have focused our attention on acquisition of the electric field components, the scanning setup and many of the described techniques may be used for magnetic field measurements utilizing a small loop antenna which would give complementary information about distribution of currents in devices under test. We believe that high resolution near-field measurements can become an attractive 
method for noninvasive investigation of functionality of microwave devices, especially during their development and testing phase when maximum information about devices and subsystems is desired.

\section{ACKNOWLEDGMENT}

This work was supported by the Science Foundation of Ireland under Contract No. 00/PI.1/C042.

${ }^{1}$ J. S. Dahele and A. L. Cullen, IEEE Trans. Microwave Theory Tech. 28, 752 (1980).

${ }^{2}$ W. Spreitzer, E. Herzer, G. Fassler, and F. M. Landstorfer, Proceedings of the COST 243 Workshop, Paderborn, 1997, p. 53.

${ }^{3}$ J. Gao, A. Lauder, Q. Ren, and I. Wolf, IEEE Trans. Microwave Theory Tech. 46, 1694 (1998).

${ }^{4}$ Y. J. Gao and I. Wolf, IEEE Trans. Microwave Theory Tech. 44, 911 (1996).

${ }^{5}$ V. Agrawal, P. Neuzil, and D. W. van der Weide, Appl. Phys. Lett. 71, 2343 (1997).

${ }^{6}$ J. D. Kraus, Antennas (McGraw-Hill, New York, 1976/1988), p. 389.
${ }^{7}$ T. Wei, X.-D. Xiang, W. G. Wallace-Friedman, and P. G. Schultz, Appl. Phys. Lett. 68, 3506 (1996).

${ }^{8}$ C. C. Wei, P. K. Wei, and W. Fann, Appl. Phys. Lett. 67, 3835 (1995).

${ }^{9}$ S. K. Dutta, C. P. Vlahacos, D. E. Steinhauer, A. S. Thanawalla, B. J. Feenstra, F. C. Wellstood, and S. M. Anlage, Appl. Phys. Lett. 74, 1999 (1999).

${ }^{10}$ A. Kramer, F. Kielmann, B. Knoll, and R. Guckenberger, Micron 27, 413 (1996).

${ }^{11}$ A. F. Lann, M. Golosovsky, and D. Davidov, Appl. Phys. Lett. 73, 2832 (1998).

${ }^{12}$ C. P. Vlahacos, D. E. Steinhauer, S. K. Dutta, B. J. Feenstra, and S. M. Angale, Appl. Phys. Lett. 72, 1778 (1998).

${ }^{13}$ F. Duever, C. Gao, I. Takeuchi, and X.-D. Xiang, Appl. Phys. Lett. 74, 2696 (1999)

${ }^{14}$ D. E. Steinhauer, C. P. Vlahacos, S. K. Dutta, F. C. Wellstood, and S. M. Anglage, Appl. Phys. Lett. 71, 1736 (1997).

${ }^{15}$ B. Knoll, F. Keilmann, A. Kramer, and R. Guckenberger, Appl. Phys. Lett. 70, 2667 (1997).

${ }^{16}$ K. Karrai and R. D. Grober, Appl. Phys. Lett. 66, 1842 (1995).

${ }^{17}$ D. P. Tsai and Y. L. Yuan, Appl. Phys. Lett. 73, 2724 (1998).

${ }^{18}$ R. Kantor, M. Lesnak, N. Berdunov, and I. V. Shvets, Appl. Surf. Sci. 144-146, 510 (1999). 